\title{
SELF-MAPS OF FLAG MANIFOLDS
}

BY

HENRY H. GLOVER AND WILLIAM D. HOMER

\begin{abstract}
Rationally, a map between flag manifolds is seen to be determined up to homotopy by the homomorphism it induces on cohomology. Two algebraic results for cohomology endomorphisms then serve (a) to determine those flag manifolds which have (nontrivial) self-maps that factor through a complex projective space, and (b) for a special class of flag manifolds, to classify the self-maps of their rationalizations up to homotopy.
\end{abstract}

0. Introduction. The homotopy classes of self-maps of a complex projective space are classified by an integral degree, and we show that, for rational homotopy, there is an analogous result for a much larger class of homogeneous spaces. These spaces are quotients of a unitary group by a closed connected subgroup of maximal rank, and, just as for projective spaces, the degree of a self-map is determined by its effect on a two-dimensional cohomology class. The first step is to observe that homotopy classes of endomorphisms of the minimal model for the rational homotopy type of such a space correspond bijectively with endomorphisms of the rational cohomology algebra (see Theorem 1.1). The second step is to classify the cohomology endomorphisms. Such a classification was given in [GH1] for the Grassmann manifold of complex $p$-planes in complex $n$-space (for $n$ greater than $2 p^{2}$ ), and it follows that the rational homotopy classes of self-maps are indeed classified by a rational degree.

Here, in Theorems 1.3 and 1.4, we give the analogous result for the flag manifolds

$$
M(1, p, q)=U(1+p+q) /(U(1) \times U(p) \times U(q)) .
$$

The cohomology endomorphisms fall into a finite number (often one) of families, each of which is classified by a rational degree. Some of these families are represented by the endomorphisms induced by conjugation in $U(1+p+q)$ by elements of the normalizer of $U(1) \times U(p) \times U(q)$, but if $1, p$, and $q$ are distinct we get only the identity in this way. The rest are represented by idempotents, which we call projective endomorphisms because they factor through the cohomology of complex projective space. Such projective endomorphisms (for any flag manifold) are in turn classified in Theorem 1.3 by factorizations over $\mathbf{Z}$ of the polynomial $1-t^{n}$.

Received by the editors March 10, 1980 and, in revised form, April 22, 1980 and August 27, 1980. 1980 Mathematics Subject Classification. Primary 55S37; Secondary 55P62, 57T15.

Key words and phrases. Flag manifold, homogeneous space, rational homotopy, self-map. 
To pass from the rational to the ordinary homotopy category, we use Theorem 1.2. It states that, given an arbitrary homomorphism between the graded rational cohomology rings of two formal finite nilpotent $\mathrm{CW}$ complexes, we can "increase its degree" (by composing with a rational automorphism, see §1) to obtain a homomorphism that is induced by a map. In particular, Theorem 1.3 determines the flag manifolds that have nontrivial projective endomorphisms on cohomology, and for each of these manifolds there are infinitely many homotopy classes of (homologically nontrivial) self-maps which factor through a complex projective space.

Finally, the flag manifolds $M(1, p, q)$ for fixed $p$ form a direct system with limit the classifying space $B(U(1) \times U(p))$. By comparing our results with those for classifying spaces given in [AM], we conclude that (if $p \neq 1$ ) among the self-maps which induce projective endomorphisms there is no infinite compatible family. This is a special case of Theorem 1.5. Thus it seems unlikely that such maps can be constructed by direct geometrical methods.

The remainder of this paper is organized as follows. In $\S 1$ we state and discuss our results, Theorems 1.1-1.5. The proof of Theorem 1. $n$ is given in section $n+1$.

We wish to thank Guido Mislin both for help in understanding the ideas from rational homotopy theory which are used in the proofs of Theorems 1.1 and 1.2 and also for specific suggestions about the formulation of those proofs. We also thank Larry Smith for prodding us into proving 1.1 in its current generality. Finally, the first author thanks Beno Eckmann and the Forschungsinstitut für Mathematik at the Eidgenössiche Technische Hochschule Zürich for their kind hospitality during the preparation of this paper.

1. Results. We consider homogeneous manifolds of the form $U(n) / H$ where $H$ is a closed, connected subgroup of maximal rank. By [BS], $H$ is conjugate to, and so we can assume equal to, $U\left(n_{1}\right) \times U\left(n_{2}\right) \times \cdots \times U\left(n_{k}\right)$ for some partition $n=n_{1}$ $+n_{2}+\cdots+n_{k}$ with $k \geqslant 2$ and $n_{1} \leqslant n_{2} \leqslant \cdots \leqslant n_{k}$. We term the quotient a (generalized) flag manifold, and denote it by $M$, or by $M\left(n_{1}, n_{2}, \ldots, n_{k}\right)$ when we wish to specify the partition. Note that all our results have immediate analogs for quaternionic flag manifolds, $\operatorname{Sp}(n) / H$, which (except for dimensions being doubled) have the same cohomology algebra structures as the corresponding complex flag manifolds.

Our first result states that the cohomology functor from the homotopy category to the category of graded algebras over the rationals is faithful on the full subcategory of rationalizations of flag manifolds.

THEOREM 1.1. Let $M_{0}$ and $M_{0}^{\prime}$ denote the rationalizations of two flag manifolds $M$ and $M^{\prime}$. Then $f \rightarrow H^{*}(f ; Q)$ induces a bijection from the set $\left[M_{0}, M_{0}^{\prime}\right]$ of homotopy classes of maps to the set of graded algebra homomorphisms from $H^{*}\left(M^{\prime} ; Q\right)$ to $H^{*}(M ; Q)$.

Recall that, since $M$ and $M^{\prime}$ are finite dimensional, each homotopy class of maps from $M_{0}$ to $M_{0}^{\prime}$ is represented by at most finitely many (if any) homotopy classes of maps from $M$ to $M^{\prime}$ [HMR]. Furthermore, there are maps from $M$ to $M^{\prime}$ whose 
rationalizations do represent infinitely many of the homotopy classes in $\left[\boldsymbol{M}_{\mathbf{0}}, \boldsymbol{M}_{0}^{\prime}\right]$. One such infinite collection is given in [Fr], and a different (though overlapping) collection is obtained from the following general result (cf. [S, 12.4]).

THEOREM 1.2. Let $X$ and $Y$ be formal nilpotent $C W$ complexes with $X$ finite and $Y$ of finite type. If $\varphi: H^{*}(Y ; Q) \rightarrow H^{*}(X ; Q)$ is a graded ring homomorphism, then there is a self-map $f: X \rightarrow X$ which induces an automorphism of $H^{*}(X ; Q)$ such that $f^{*} \circ \varphi$ is induced by a map $g: X \rightarrow Y$.

We next investigate the cohomology auto- and endomorphisms of flag manifolds. For any graded algebra over $Q$ and any $\lambda \in Q$, the formula $\varphi(x)=\lambda^{\operatorname{dim}(x)} x$, for all homogeneous $x$, gives a well-defined automorphism. We refer to these as "grading" endomorphisms. (In [O] and [GH1], they were termed Adams endomorphisms, by analogy with the Adams operations on $B U(n)$.) Since our cohomology algebras are concentrated in even dimensions, we can use $\frac{1}{2} \operatorname{dim}(x)$ as the exponent of $\lambda$. There are corresponding self-maps of $M$, for infinitely many $\lambda \in \mathbf{Z}$, by [Fr]. The nontrivial grading endomorphisms form a group $\mathcal{G}$ in the center of the monoid of all nontrivial endomorphisms, so cosets of $\mathcal{G}$ are well defined. Since for a flag manifold $M, H^{*}(M ; Z)$ is free and finitely generated, there is a corresponding coset structure for endomorphisms of it modulo the submonoid of grading endomorphisms with degree $\lambda \in \mathbf{Z}$. For both coefficient rings, this coset structure will provide a notion of degree for all cohomology endomorphisms.

There are also self-maps which have direct constructions. Conjugation by an element of $N(H)$, the normalizer of $H$ in $U(n)$, induces a self-map of $M$. Nontrivial examples require $N(H) / H \neq 1$, that is, $n_{i}=n_{j}$ for a distinct pair of subscripts. In what follows, we use the term "conjugation" to describe both a self-map of this kind (including the identity) and also the induced automorphism of cohomology.

For all flag manifolds for which results are available, the automorphism group of the rational cohomology algebra is precisely $\mathcal{G} \times(N(H) / H)$ when $n \geqslant 3$ and $\mathcal{G}$ when $n=2$. These results include the manifolds coming from partitions of the form $n=1+\cdots+1$ [L3], [EL], $n=1+\cdots+1+q$ with $q \geqslant \frac{1}{2} n$ [L1], [L2], $n=2+q[\mathbf{O}]$, and $n=p+q$ with $q>p$ [GH1], [Br]. The Grassmann manifolds, $n=p+q$, are noteworthy because in all cases for which the entire monoid of endomorphisms has been determined, it consists simply of the automorphisms, $\mathcal{G} \times(N(H) / H)$, together with the zero endomorphism. Known cases include $p \leqslant 3$ [O], [GH1] and $q \geqslant 2 p^{2}-p+1$ [GH1]. For more general flag manifolds, there can be other types of endomorphisms, as we see in Theorem 1.3.

Notice that for a flag manifold $M$ for which the cohomology automorphism group is $\mathcal{G} \times(N(H) / H)$ (and this may not be a restriction), we obtain from 1.2 the corollary: Given a nonzero endomorphism of $H^{*}(M ; Q)$, we can increase its degree by composing with a grading endomorphism with degree a positive integer to obtain an endomorphism which is induced by a self-map. In particular, for every prime $p$, there is an integer $\lambda$ divisible by $p$ so that the grading endomorphism of degree $\lambda$ is induced by a self-map. This gives examples in addition to those constructed in $[\mathbf{F r}]$. 
In this paper we consider partitions with $n_{i}=1$ for (at least) $i=1$. For each such $i$, there is a natural map $M \rightarrow M(1, n-1)=C P(n-1)$ which induces a monomorphism of cohomology algebras. A cohomology endomorphism which factors through such a monomorphism we call "projective". Since any such monomorphism can be obtained from any other by composing with the appropriate conjugation, we focus on $i=1$. To describe projective endomorphisms in more detail, we recall (from $[\mathrm{B}]$ ) that $H^{*}(M ; Q)$ is generated as an algebra by classes $\left\{x_{i, j}\right.$ : $\left.1 \leqslant i \leqslant k, 1 \leqslant j \leqslant n_{i}\right\}$, where $x_{i, j}$ has dimension $2 j$. For $n_{1}=1, x_{1,1}$ generates the subalgebra which is the image of $H^{*}(C P(n-1) ; Q)$ under the natural monomorphism. Our next result characterizes projective endomorphisms in terms of cyclotomic polynomials. Using 1.2, we obtain examples of self-maps of $M$ which factor through $C P(n-1)$.

THEOREM 1.3. Given a factorization of $1-t^{n}$ in the polynomial ring $\mathbf{Z}[t], 1-t^{n}$ $=P_{1}(t) P_{2}(t) \ldots P_{k}(t)$, let $n_{i}$ denote the degree of $P_{i}(t)$. If $P_{1}(t)=1-t$ and $\lambda$ is any rational number, then the formula

$$
\varphi\left(1+x_{i, 1}+x_{i, 2}+\cdots+x_{i, n_{i}}\right)=P_{i}\left(\lambda x_{1,1}\right), \quad 1 \leqslant i \leqslant k,
$$

gives a well-defined (projective) endomorphism of $H^{*}\left(M\left(1, n_{2}, \ldots, n_{k}\right) ; Q\right)$. Conversely, every nonzero projective endomorphism (for any flag manifold with $n_{1}=1$ ) has this form, up to a conjugation.

There is an analogous statement for integral cohomology, with $\lambda$ required to be an integer.

When the partition has length $k=3, n=1+p+q$, we can show, for many values of $p$ and $q$, that every coset of $\mathcal{G}$ is represented by a conjugation or a projective endomorphism. When $p=1$, this follows for all $q \geqslant 1$ from [EL]. Notice that in this case nonzero projective endomorphisms exist if and only if $q$ is even. If $1<p<q$, then the only conjugation is the identity, and we have the following result.

THEOREM 1.4. If $1, p$, and $q$ are distinct positive integers and $q>2 p^{2}-1$, then every ( graded algebra) endomorphism of $H^{*}(M(1, p, q) ; R), R=\mathbf{Z}$ or $Q$, is either $a$ grading endomorphism or a projective endomorphism.

The restriction $q \gg p$ arises from our method of proof and we conjecture that the result is true without it. Again, for many values of $p$ and $q$, there are no nonzero projective endomorphisms. For example, if $p=2$, then by 1.3 a nonzero projective endomorphism exists if and only if $n=1+2+q$ is congruent to zero modulo 3 or 4.

As before, the automorphism group (over the rationals) is $\mathcal{G} \times(N(H) / H)$, which is just $\mathcal{G}$ in the cases covered by the theorem. Including the $p=1$ case, the following somewhat sharper statement is immediate from Theorems 1.3 and 1.4. In the monoid of nonzero endomorphisms, a complete set of left coset representatives for the automorphism group, $\mathcal{G} \times(N(H) / H)$, consists of the identity and finitely many projective endomorphisms $\varphi$ with $\varphi\left(x_{1,1}\right)=x_{1,1}$, which are idempotents. We conjecture that an analogous result is true for an arbitrary flag manifold. 
We next compare our results with those for classifying spaces of Lie groups [AM] and conclude with a comment on the limitations of direct geometrical constructions. If we fix $n_{1}, n_{2}, \ldots, n_{k-1}$ and let $n_{k}$ range over (any infinite subset of) the positive integers, we obtain a nested family of flag manifolds with direct limit $B G$, where $G=U\left(n_{1}\right) \times \cdots \times U\left(n_{k-1}\right)$. Now $\left\{x_{k, i}: 1 \leqslant i \leqslant n_{k}\right\}$ are redundant generators for $H^{*}\left(M\left(n_{1}, \ldots, n_{k}\right) ; \mathbf{Z}\right)$, and the remaining generators give, in the inverse limit, classes which freely generate $H^{*}(B G ; \mathbf{Z})$.

Since $H^{*}(B G ; \mathbf{Z})$ is free, all of the endomorphisms we have found for flag manifolds correspond to (a rather small subset of the) cohomology endomorphisms of $B G$, and we shall carry over our terminology. Furthermore, these endomorphisms of $H^{*}(B G ; \mathbf{Z})$ are actually inverse limits of cohomology endomorphisms of flag manifolds. This is clear for conjugations and grading endomorphisms, and given a projective endomorphism described as in Theorem 1.3, we can take

$$
n_{k}^{(0)}=n_{k}, \quad n_{k}^{(1)}=n_{k}+n, \quad n_{k}^{(2)}=n_{k}+2 n, \ldots
$$

and

$$
P_{k}^{(0)}(t)=P_{k}(t), \quad P_{k}^{(1)}(t)=\left(1+t^{n}\right) P_{k}(t), \quad P_{k}^{(2)}(t)=\left(1+t^{n}+t^{2 n}\right) P_{k}(t), \ldots,
$$

to get a compatible family.

It is natural to ask if an analogous statement can be made for self-maps. For conjugations, the answer is clearly yes: A conjugation of $B G$ is the direct limit of conjugations of flag manifolds. For grading endomorphisms, the answer is not clear: Neither Theorem 1.2 nor [Fr] definitely produce infinite families of self-maps which are compatible with respect to inclusions and induce grading endomorphisms on cohomology. For projective endomorphisms, the answer is, with very few exceptions, no. The precise statement is as follows.

THEOREM 1.5. Let $G=U(1) \times U\left(n_{2}\right) \times \cdots \times U\left(n_{k-1}\right)$, with $1 \leqslant n_{2} \leqslant \cdots \leqslant$ $n_{k-1}$. If there is an integer $n_{k} \geqslant n_{k-1}$ and a self-map of $B G$ which restricts to $a$ self-map $g$ of $M\left(1, n_{2}, \ldots, n_{k}\right)$ so that $g^{*}$ is a nonzero projective endomorphism of cohomology, then $G$ is $U(1)$ or $U(1) \times U(1)$. Thus, except on $\{C P(m)\}$ or $\{M(1,1,2 m)\}$, there can be no infinite compatible family of self-maps of flag manifolds which induce projective endomorphisms of cohomology.

Note that the same statement holds for self-maps defined after localization which inverts a fixed finite set of primes.

If we exclude complex projective spaces, the known direct geometrical constructions for self-maps of flag manifolds include those for conjugations and for the fixed point free self-maps of [GH2], and they do produce infinite compatible families of self-maps. Theorem 1.5 may thus be interpreted as negative evidence for the existence of similarly nice direct constructions for the self-maps (known to exist by Theorem 1.2) which induce projective endomorphisms. The flag manifolds of the form $M(1,1,2 q)$ are an exception because the idea for the familiar construction of a unit normal vector field on an odd-dimensional sphere also gives a construction for compatible self-maps inducing projective endomorphisms of degree 1 on such flag manifolds. See [GHS] for more details. 
2. Proof of Theorem 1.1. We begin by recalling from [B] the algebra structure of $H^{*}(M ; Q)$. Let $S_{i}=\left\{(i, j) \in \mathbf{Z} \times \mathbf{Z}: 1 \leqslant j \leqslant n_{i}\right\}$ and $S=\cup\left\{S_{i}: 1 \leqslant i \leqslant k\right\}$. Give $A=Q\left[x_{\alpha}: \alpha \in S\right]$ a grading by setting the dimension of $x_{i, j}$ equal to $2 j$, and taking $A^{m}$ to be the span of all monomials of total dimension $m$. Write

$$
\prod_{i=1}^{k}\left(1+\sum\left\{x_{\alpha}: \alpha \in S_{i}\right\}\right)=1+R_{1}+R_{2}+\cdots+R_{n}
$$

where $R_{m}$ has dimension $2 m$. Let $I$ be the ideal in $A$ generated by $R_{1}, \ldots, R_{n}$. Then $A / I$ is isomorphic as a graded algebra to $H^{*}(M ; Q)$, with $x_{i, j}$ corresponding to the $j$ th Chern class of the canonical complex $n_{i}$-plane bundle over $M$. This description follows from the fact that the Serre spectral sequence for the fibration $U(n) / H \rightarrow B H \rightarrow B U(n)$ collapses, since both the base and the fiber have nontrivial cohomology only in even dimensions. (Thus the description for $\mathbf{Z}$ coefficients is analogous.)

For any integer $i, 1 \leqslant i \leqslant k$, the generators $\left\{x_{\alpha}: \alpha \in S_{i}\right\}$ are redundant, as one sees by inductively solving $R_{j}=0$ for $x_{i, j}$ in terms of $\left\{x_{\alpha}: \alpha \in S-S_{i}\right\}$, for $j=1,2, \ldots, n_{i}$. We shall take $i=k$ to get a presentation of $H^{*}(M ; Q)$ with the fewest generators. Then if $B=Q\left[x_{\alpha}: \alpha \in S-S_{k}\right]$ and $J$ is the kernel of the epimorphism $B \rightarrow A / I$, it follows that $H^{*}(M ; Q)$ is isomorphic to $B / J$. One set of generators for the ideal $J$ can be obtained by expressing $\left\{R_{m}: n_{k}+1<m \leqslant n\right\}$ in terms of $\left\{x_{\alpha}: \alpha \in S-S_{k}\right\}$, but it will be more convenient to use another set, defined as follows. In the graded formal power series ring, $Q\left[\left[x_{\alpha}: \alpha \in S-S_{k}\right]\right]$, write

$$
\left[\prod_{i=1}^{k-1}\left(1+\sum\left\{x_{\alpha}: \alpha \in S_{i}\right\}\right)\right]^{-1}=1+T_{1}+T_{2}+\ldots
$$

where $T_{m}$ has dimension $2 m$. It is easy to verify that the ideal in $B$ generated by $\left\{T_{m}: n_{k}+1 \leqslant m \leqslant n\right\}$ is $J$.

The rest of the proof consists of observing that these presentations of their cohomology algebras put flag manifolds within the scope of the methods of $\$ 16$ of [BG]. Recall that a morphism of DG (differential graded) algebras is said to be a weak equivalence if it induces an isomorphism on homology. A minimal model for a space $X$ is a minimal free commutative (in the graded sense) DG algebra $M X$ together with a weak equivalence from $M X$ to $A X$, the polynomial de Rham complex of $X$. If $X$ is simply connected, such an algebra $M X$ will be minimal if the image of the differential is contained in the decomposables. A space $X$ is said to be formal if there is also a weak equivalence from $M X$ to $H^{*}(X ; Q)$, where the latter is equipped with the zero differential.

Following [BG], we can give a simple construction for a minimal model $C$ for a flag manifold $M$. Let $C$ be the free commutative algebra over $Q$ with generators $\left\{x_{\alpha}: \alpha \in S-S_{k}\right\}$ and $\left\{y_{m}: n_{k}+1 \leqslant m \leqslant n\right\}$ and with differential $d$ of degree +1 such that $d x_{\alpha}=0$ and $d y_{m}=T_{m}$. Here $x_{\alpha}$ has the same dimension as in $B$, which we regard as a subalgebra of $C$, and so $y_{m}$ has dimension $2 m-1$. Then the obvious projection $\pi: C \rightarrow B / J=H^{*}(M ; Q)$ is a weak equivalence which can be 
lifted to a weak equivalence from $C$ to the polynomial de Rham complex $A M$ of $M$. The key point in this construction is that, by 3.9 of [Ba], the expressions $\left\{T_{m}\right.$ : $n_{k}+1 \leqslant m \leqslant n$ \} form an ESP (or $E$-) sequence, which is to say that $T_{m}$ does not represent a zero divisor in $B /\left\langle T_{n_{k}+1}, \ldots, T_{m-1}\right\rangle$ for $m=n_{k}+1, \ldots, n$.

Now by 6.4 and 7.5 of [BG], the weak equivalence $\pi: C \rightarrow B / J$ induces a bijection of homotopy classes of morphisms $\pi_{*}:\left[C^{\prime}, C\right] \rightarrow\left[C^{\prime}, B / J\right]$. But the homotopy relation in $\operatorname{Mor}\left(C^{\prime}, B / J\right)$ is trivial: Suppose $\psi_{1} \sim \psi_{2}$. Then they induce the same homomorphism on homology, which we can regard as a homomorphism $\varphi: B^{\prime} / J^{\prime} \rightarrow B / J$. Since as a DGA with zero differential $B / J$ equals $H_{*}(B / J)$, we must have $\psi_{1}\left(x_{\alpha}^{\prime}\right)=\varphi\left(x_{\alpha}^{\prime}\right)=\psi_{2}\left(x_{\alpha}^{\prime}\right)$. Since $B / J$ is zero in odd dimensions, we must have $\psi_{1}\left(y_{m}^{\prime}\right)=0=\psi_{2}\left(y_{m}^{\prime}\right)$. Hence $\psi_{1}=\varphi \circ \pi^{\prime}=\psi_{2}$, and it follows that $\pi^{\prime}$ gives a bijection $\pi^{\prime *}$ from the set of homomorphisms from $B^{\prime} / J^{\prime}$ to $B / J$ to the set $\left[C^{\prime}, B / J\right]$, which is just the set of morphisms from $C^{\prime}$ to $B / J$.

The bijection $\left(\pi^{\prime *}\right)^{-1} \circ \pi_{*}:\left[C^{\prime}, C\right] \rightarrow \operatorname{Hom}\left(B^{\prime} / J^{\prime}, B / J\right)$ takes a class of morphisms to the induced homomorphism on homology, and Theorem 1.1 now follows from the Sullivan-de Rham Equivalence Theorem: the homotopy category of minimal algebras over $Q$ of finite type is equivalent to the homotopy category of connected nilpotent rational Kan complexes of finite type.

REMARK. In some cases, the homotopy relation in $\operatorname{Mor}\left(C^{\prime}, C\right)$ is also trivial. By [Ba] or a simple counting argument, the cocycle with the smallest odd dimension in $C$ is $d\left(y_{r} y_{r+1}\right)$, where $r=n_{k}+1$, which has dimension $4 n_{k}+5$. Thus if $2 n^{\prime}-1<$ $4 n_{k}+5$, or more simply $n^{\prime}<2 n_{k}+3$, a morphism from $C^{\prime}$ to $C$ is uniquely determined by the homomorphism it induces on homology.

3. Proof of Theorem 1.2. We continue to use the notation for minimal models introduced in the last section. By 6.4 and 7.5 of [BG], there is a morphism $\varphi^{\prime}$ of DG algebras which makes the following diagram commute up to homotopy.

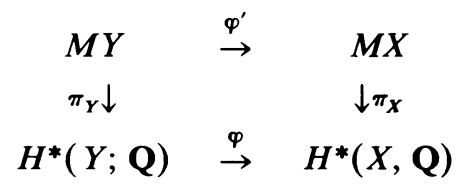

By the Sullivan-de Rham Equivalence Theorem (see [S] or [BG]), there is a corresponding map $\varphi^{\prime \prime}: X_{0} \rightarrow Y_{0}$ between rationalizations of $X$ and $Y$. We now show that for some choice of $f: X \rightarrow X$, we can fill in the dotted arrow to make the following diagram homotopy commute (cf. [GHM]).

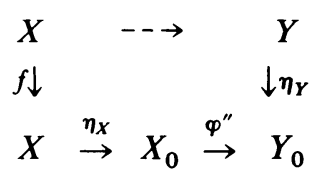

The localization map $\eta_{Y}$ can be factored into a sequence of principal fibrations $\eta_{i}$ with fibers $K\left(G_{i}, m_{i}\right)$, where $G_{i}$ is a torsion group and $m_{i}<m_{i+1}$. Let $f_{1}$ be the identity on $X$ and suppose inductively that $\varphi^{\prime \prime} \circ \eta_{X} \circ f_{i}$ lifts to the base space of $\eta_{i}$. The obstruction to lifting to the total space is an element $u$ of $H^{m_{i}+1}\left(X ; G_{i}\right)$ and so has finite order. Since $X$ is formal, there is a self-map $h$ of $X$ so that $h$ induces an 
isomorphism on rational cohomology and also $h^{*} u=0$ (see 12.2 of [S]). If we put $f_{i+1}=f_{i} \circ h$, then $\varphi^{\prime \prime} \circ \eta_{X} \circ f_{i+1}$ lifts to the total space of $\eta_{i}$, which is the base space of $\eta_{i+1}$. After finitely many steps, we reach an $i$ for which $m_{i}$ is greater than the dimension of $X$. Then for $f=f_{i}$, there are no obstructions to lifting $\varphi^{\prime \prime} \circ \eta_{X} \circ f$ to $Y$.

4. Proof of Theorem 1.3. Suppose $(U(1))^{n} \subseteq H \subseteq H^{\prime} \subseteq U(n)$, and let $M=$ $U(n) / H$ and $M^{\prime}=U(n) / H^{\prime}$ be the corresponding flag manifolds. There are canonical maps $B i: B H \rightarrow B H^{\prime}$ and $\pi: M \rightarrow M^{\prime}$ which both induce monomorphisms of cohomology rings (since the Serre spectral sequences of the relevant fibrations collapse). Thus if $B / J$ and $B^{\prime} / J^{\prime}$ are the presentations described in $\$ 2$ for $H^{*}(M ; \mathbf{Z})$ and $H^{*}\left(M^{\prime} ; \mathbf{Z}\right)$, it follows that we can regard $B^{\prime}$ as a subalgebra of $B$ and that $J^{\prime}=J \cap B^{\prime}$. For the case $M=M(1, \ldots)$ and $M^{\prime}=M(1, n-1)$, we can restate this as follows.

Proposition 4.1. In $H^{*}(M(1, \ldots) ; Q), x_{1,1}^{n}$ is the smallest power of $x_{1,1}$ which is zero.

To prove the first assertion of Theorem 1.3, suppose we are given the factorization of $1-t^{n}$ and define an endomorphism $\varphi$ of $A$ by the given formula. We must show $\varphi$ preserves the ideal $I=\left\langle R_{1}, \ldots, R_{n}\right\rangle$. To simplify notation, identify $t$ and $x_{1,1}$. If we apply $\varphi$ to the defining equation for the $R_{m}$, we have $P_{1}(\lambda t) P_{2}(\lambda t) \ldots P_{k}(\lambda t)=\sum \varphi\left(R_{m}\right)$. But the left side is just $1-(\lambda t)^{n}$. Hence $\varphi\left(R_{s}\right)$ $=0$ if $1 \leqslant s \leqslant n-1$ and $\varphi\left(R_{n}\right)=-\lambda^{n} t^{n} \in I$, by 4.1. Thus $\varphi(I) \subseteq I$.

Conversely, suppose $\varphi$ is a nonzero projective endomorphism of $A / I$. By definition of $I$, we have

$$
(1+t)^{-1}=\prod_{i=2}^{k}\left(1+x_{i, 1}+\cdots+x_{i, n_{i}}\right) \text { modulo } I .
$$

If we apply $\varphi$ to both sides, we have, by 4.1 ,

$$
(1-\lambda t)^{-1}=\prod_{i=2}^{k} Q_{i}(t) \quad \text { modulo }\left\langle t^{n}\right\rangle .
$$

Here $\lambda$ is defined by $\varphi(t)=-\lambda t$ and $Q_{i}(t)$ is a polynomial of degree $n_{i}$ in $t$. If we put $P_{i}(t)=Q_{i}(t / \lambda)$, we have

$$
1+t+t^{2}+\cdots+t^{n-1}=\prod_{i=2}^{k} P_{i}(t) .
$$

Multiplying both sides by $P_{1}(t)=1-t$ gives the desired factorization of $1-t^{n}$.

5. Proof of Theorem 1.4. We begin with a cautionary remark. Since there is a classification of cohomology endomorphisms for many Grassmann manifolds [GH1], which correspond to partitions of length 2, and (at least for automorphisms) for the flag manifolds corresponding to the partitions $n=1+\cdots+1$ [L1], we might hope to proceed inductively by refining the first type of partition or coarsening the second. 
In general, if $M$ corresponds to a partition which is a refinement of that for $M^{\prime}$, recall from $\S 4$ that $\pi^{*}: H^{*}\left(M^{\prime}\right) \rightarrow H^{*}(M)$ is a monomorphism. Given an endomorphism of $H^{*}(M)$, can we restrict it to an endomorphism of $H^{*}\left(M^{\prime}\right)$ ? The conjugations show that this is not possible in general. Conversely, given an endomorphism of $H^{*}\left(M^{\prime}\right)$, can we extend it to an endomorphism of $H^{*}(M)$ ? Both conjugations and projective endomorphisms show that this also is not possible in general. For example, the factorization of $1-t^{n}$ into irreducible (i.e. cyclotomic) polynomials corresponds, by Theorem 1.3, to a projective endomorphism which cannot be so extended.

Note. The arguments in this section work equally well for cohomology with rational or integral coefficients, and we use the integers.

Case I. $\varphi\left(T_{q+1}\right) \neq 0$.

We begin by taking advantage of a result of [EL] to shorten the proof given in a preliminary version, [GH1], of this paper. Let $\varphi$ be a graded ring endomorphism of $H^{*}(M(1, p, q) ; \mathbf{Z})$. For simplicity, let $t$ denote $x_{1,1}$ and let $x_{j}$ denote $x_{2, j}$ for $1 \leqslant j \leqslant p$.

LEMMA 5.1. Let $\lambda$ denote the coefficient of $x_{1}$ in $\varphi\left(x_{1}\right)$. If $\varphi\left(T_{q+1}\right) \neq 0$ and $q \geqslant 2 p^{2}-2$, then the coefficient of $x_{j}$ in $\varphi\left(x_{j}\right)$ is $\lambda^{j}$ and the coefficient of $x_{1} x_{p-1}$ in $\varphi\left(x_{p}\right)$ is zero.

Proof. By [EL], the coefficient of $x_{1}$ in $\varphi(t)$ is zero. Given this fact, the proof proceeds as in paragraphs 1 through 5 in section 4 of [GH1]. We also use Lemma 5.2 below in place of Proposition 1 of [GH1].

Thus under the hypotheses of Lemma 5.1, $\varphi$ has the form

$$
\begin{aligned}
\varphi(t) & =a t, \\
\varphi\left(x_{1}\right) & =\lambda x_{1}+b_{1} t \\
\varphi\left(x_{2}\right) & =\lambda^{2} x_{2}+b_{2} t^{2}+\ldots \\
& \ldots \\
\varphi\left(x_{p}\right) & =\lambda^{p} x_{p}+b_{p} t^{p}+c t x_{p-1}+0 x_{1} x_{p-1}+\ldots
\end{aligned}
$$

The idea for the rest of the argument is, as in [GH1], to compare coefficients in two expressions for $\varphi\left(T_{q+1}\right)$. On one hand, $\varphi(J) \subseteq J$ implies that $\varphi\left(T_{q+1}\right)=N T_{q+1}$ for some integer $N$. On the other hand, we can apply the definition of $\varphi$ given above in terms of the coefficients $\lambda, a, b_{1}, \ldots$, to an explicit formula for $T_{q+1}$. We carry out the derivation of this formula far enough to be able to read off the coefficients of specific monomials. From the definition,

$$
\sum T_{m}=\left[(1+t)\left(1+x_{1}+\cdots+x_{p}\right)\right]^{-1}=\left[\sum(-t)^{i}\right]\left[\sum\left(-x_{1}-\cdots-x_{p}\right)^{j}\right] \text {. }
$$

In expanding powers of $-\left(x_{1}+\cdots+x_{p}\right)$, we shall use for the multinomial coefficients the notation

$$
\left(m_{1}, \ldots, m_{p}\right)=(-1)^{m_{1}+\cdots+m_{p}} \frac{\left(m_{1}+\cdots+m_{p}\right) !}{\left(m_{1} !\right) \ldots\left(m_{p} !\right)} .
$$

We now assume $p \geqslant 3$. If $p=2$, the argument is similar in spirit to what follows, but different in detail. 
We can find a monomial of the form $x_{p-1}^{i} x_{p}^{j}$ in dimension $2(q+1)$ precisely when we can write $q+1=i(p-1)+j p$ for two nonnegative integers $i$ and $j$. For this and similar requirements, the following lemma provides a simple sufficient condition. The proof is an easy exercise.

LEMMA 5.2. If $m \geqslant p(p-2)+i_{0}(p-1)+j_{0} p$, then we can write $m=i(p-1)$ $+j p$ with $i \geqslant i_{0}$ and $j \geqslant j_{0}$.

Thus if $q+1 \geqslant p(p-2)$, we can compare coefficients of a monomial of the form $x_{p-1}^{i} x_{p}^{j}$ in the two expressions for $\varphi\left(T_{q+1}\right)$ to find that $(i, j) N=(i, j) \lambda^{q+1}$, so $N=\lambda^{q+1}$.

LEMMA 5.3. If $q \geqslant 2 p^{2}-1$, then $a=\lambda$ and $b_{1}=c=0$ in (1).

Proof. The hypothesis on $q$ guarantees that monomials of the forms we require can be found in dimension $2(q+1)$. Comparison of coefficients of a monomial of the form $t x_{p-1}^{i} x_{p}^{j}$ gives

$$
-(i, j) \lambda^{q+1}=-(i, j) a \lambda^{q}+(1, i, j) b_{1} \lambda^{q}+(i-1, j+1)(j+1) c \lambda^{q-p+1} .
$$

The terms on the right are the contributions of the images under $\varphi$ of $t x_{p-1}^{i} x_{p}^{j}$, $x_{1} x_{p-1}^{i} x_{p}^{j}$, and $x_{p-1}^{i-1} x_{p}^{j+1}$ to the total coefficient of $t x_{p-1}^{i} x_{p}^{j}$ in $\varphi\left(T_{q+1}\right)$. The multinomial coefficients are the coefficients of these monomials in $T_{q+1}$ and the factor $(j+1)$ in the third term comes from the expansion of

$$
\left[\varphi\left(x_{p}\right)\right]^{j+1}=\left[\lambda^{p} x_{p}+c t x_{p-1}+\ldots\right]^{j+1} \text {. }
$$

The equation simplifies to

$$
\lambda^{p}=a \lambda^{p-1}+(1+i+j) b_{1} \lambda^{p-1}-i c .
$$

If $j \geqslant p-1$, from $t x_{p-1}^{i+p} x_{p}^{j-p+1}$ we get an equation which can be obtained from (2) by substituting $i+p$ for $i$ and $j-p+1$ for $j$.

$$
\lambda^{p}=a \lambda^{p-1}+(2+i+j) b_{1} \lambda^{p-1}-(i+p) c .
$$

Eliminate first $a$ and then $b$, from (2) and (3).

$$
\begin{gathered}
0=b_{1} \lambda^{p-1}-p c, \\
\lambda^{p}=a \lambda^{p-1}+(p+q) c .
\end{gathered}
$$

Similarly, from monomials $t^{2} x_{p-1}^{i} x_{p}^{j}$ and $t^{2} x_{p-1}^{i+p} x_{p}^{j-p+1}$, we get a pair of equations involving an additional coefficient, $b_{2}$. Eliminating $b_{2}$ and substituting from (4) and (5) gives $0=c\left[(p+q) c-2 \lambda^{p}\right]$. If $c \neq 0$, then

$$
(p+q) c=2 \lambda^{p},
$$

which leads to a contradiction. From $t^{3} x_{p-1}^{i} x_{p}^{j}$ and $t^{3} x_{p-1}^{i+p} x_{p}^{j-p+1}$, we get another pair of equations involving $b_{3}$ as well as the previous coefficients. Eliminating $b_{3}$ and then $b_{2}$ and substituting from (4), (5), and (6) yields $c=0$, the desired contradiction. (The reader is warned that the details require a little perseverance. Each of the last pair of equations involves fifteen terms.)

Since $c=0$, it follows from (4) and (5) that $b_{1}=0$ and $a=\lambda$. This concludes the proof of Lemma 5.3. 
LEMMA 5.4. If $a=\lambda$ and $b_{1}=c=0$ in (1), then $\varphi$ is the grading endomorphism of degree $\lambda$, provided $q \geqslant p^{2}-p-1$.

Proof. Write all monomials involved in (1) in the standard form $t^{j} x_{1}^{i_{1}} \ldots x_{p}^{i_{j}}$. Exclude $t, x_{1}, x_{2}, \ldots, x_{p}, t x_{p-1}, x_{1} x_{p}$, and order the rest lexicographically by exponents. If we assume inductively that all monomials less than a given monomial have coefficient zero in (1), and compare coefficients of that monomial in the two expressions for $\varphi\left(T_{q+1}\right)$, then we see that it also has coefficient zero in (1) (cf. paragraph 6, $\$ 4$ of [GH1]).

Case II. $\varphi\left(T_{q+1}\right)=0$.

LEMMA 5.5. If $q \geqslant 2 p^{2}-2 p-1$, and $\varphi\left(T_{q+1}\right)=0$, then $\varphi\left(T_{j}\right)=0$ for $q+2<$ $j \leqslant q+p=n-1$.

Proof. The argument is a straightforward generalization of the proof of Lemma $2, \S 4$ of [GH1]. Again there is an induction on monomials ordered lexicographically by exponent. As in the proof of Lemma 5.3, the hypothesis on $q$ guarantees that convenient monomials can be found in the relevant dimensions.

LEMMA 5.6. If $\varphi(t)=$ at and $\varphi\left(T_{j}\right)=0$ for $q+1 \leqslant j<n-1$, then $\varphi$ is a projective endomorphism.

Proof. Since $\left(1+x_{1}+\cdots+x_{p}\right) \sum T_{j}=\Sigma(-t)^{j}$, it follows from the hypothesis that $\varphi\left(T_{n}\right)=\varphi\left((-t)^{n}\right)=(-a t)^{n}$. Let $P=(1+t)\left(1+x_{1}+\cdots+x_{p}\right)$. Applying $\varphi$ to the defining equation $P^{-1}=\Sigma T_{j}$ gives

$$
[\varphi(P)]^{-1}=Q \sum_{j}(-a t)^{n j}=Q\left[1-(-a t)^{n}\right]^{-1},
$$

where $Q$ is a polynomial of total degree $q$. Then $Q \varphi(P)=1-(-a t)^{n}$ is a factorization of $1-(-a t)^{n}$ in $\mathbf{Z}\left[t, x_{1}, \ldots, x_{p}\right]$ and hence in $\mathbf{Z}[t]$. In particular, $\varphi(P)$ is a polynomial in $t$, so that $\varphi$ is projective.

Finally, of the four requirements on $q, q>2 p^{2}-1$ dominates the hypotheses of Lemmas 5.1-5.5.

6. Proof of Theorem 1.5. In this section we consider self-maps of a space $X$ which are defined on any localization of $X$ which inverts only finitely many primes. We shall suppress the localization in the notation and simply refer to these as self-maps of $X$.

Let $f$ be such a self-map of $B G$, where $G=U(1) \times U\left(n_{2}\right) \times \cdots \times U\left(n_{k-1}\right)$, and let $T$ be a maximal torus in $G$. By [AM], there is a conjugate $T^{\prime}$ of $T$ and an endomorphism $\psi$ so that the following diagram commutes.

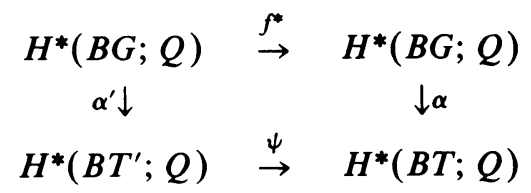

Here $\alpha$ and $\alpha^{\prime}$ are induced by the inclusion maps. Recall that, for $1<i<k-1$, $\alpha^{\prime}\left(1+x_{i, 1}+\cdots+x_{i, n_{i}}\right)$ splits into linear factors $\left(1+t_{i, 1}^{\prime}\right)\left(1+t_{i, 2}^{\prime}\right) \ldots\left(1+t_{i, n_{i}}^{\prime}\right)$ 
and that the two-dimensional classes $\left\{t_{i, j}^{\prime}: 1 \leqslant i \leqslant k-1,1 \leqslant j \leqslant n_{i}\right\}$ freely generate $H^{*}\left(B T^{\prime} ; Q\right)$ as an algebra over $Q$. The analogous statements hold for $\alpha$ and $B T$.

Now suppose that, for some $n_{k} \geqslant n_{k-1}, f$ restricts to a self-map $g$ of (the corresponding localization of $M\left(1, n_{2}, \ldots, n_{k}\right)$ and that $g^{*}$ is a nonzero projective endomorphism. Then, by $1.3, g^{*}$ and also $f^{*}$ is determined by a nonzero $\lambda \in Q$ and a factorization $1-z^{n}=P_{1}(z) \ldots P_{k}(z)$ where $n=1+n_{2}+\cdots+n_{k}$. It suffices to take $\lambda=1$. Then

$$
\begin{aligned}
P_{i}\left(t_{1,1}\right) & =\alpha\left(P_{i}\left(x_{1,1}\right)\right)=\alpha \circ f^{*}\left(1+x_{i, 1}+\ldots\right) \\
& =\psi \circ \alpha^{\prime}\left(1+x_{i, 1}+\ldots\right)=\psi\left(1+t_{i, 1}^{\prime}\right) \ldots\left(1+t_{i, n_{i}}^{\prime}\right) .
\end{aligned}
$$

Since $t_{1,1}$ has cup length $n-1$, it follows that the polynomial $P_{i}(z)$ splits into linear factors in $Q[z]$, for $1 \leqslant i \leqslant k-1$. But this is possible only if $k=2$ (since $\left.P_{1}(z)=1-z\right)$ or if $k=3$ and $P_{2}(z)=1+z$, in which case $n_{2}=1$.

\section{REFERENCES}

[AM] J. F. Adams and Z. Mahmud, Maps between classifying spaces, Invent. Math. 35 (1976), 1-41.

[Ba] P. F. Baum, On the cohomology of homogeneous spaces, Topology 7 (1968), 15-38.

[BG] A. K. Bousfield and V. K. A. M. Gugenheim, On PL de Rham theory and rational homotopy type, Mem. Amer. Math. Soc. No. 179 (1976).

[B] A. Borel, Topics in the homology theory of fiber bundles, Lecture Notes in Math., vol. 36, Springer-Verlag, Berlin and New York, 1967.

[BS] A. Borel and J. de Siebenthal, Les sous-groupes fermes de rang maximum des groupes de Lie clos, Comment. Math. Helv. 23 (1949), 200-221.

[Br] S. Brewster, Automorphisms of the cohomology ring of finite Grassmann manifolds, Ph. D. Dissertation, Ohio State Univ., Columbus, 1978.

[EL] J. Ewing and A. Liulevicius, Homotopy rigidity of linear actions on friendly homogeneous spaces, J. Pure Appl. Algebra 18 (1980), 259-267.

[Fr] E. Friedlander, Maps between localized homogeneous spaces, Topology 16 (1977), 205-216.

[GH1] H. Glover and W. Homer, Endomorphisms of the cohomology ring of finite Grassmann manifolds (Proc. Northwestern Univ. Conf. Geometric Applications of Homotopy Theory), Lecture Notes in Math., vol. 657, Springer-Verlag, Berlin and New York, 1978, pp. 170-193.

[GH2] __ Fixed points on flag manifolds (preprint).

[GH3] Cohomology endomorphisms of flag manifolds. I, II, 1978, (Preliminary report).

[GHM] H. Glover, W. Homer and G. Mislin, Immersions in manifolds of positive weights, Algebraic Topology (Proc. Conf., Vancouver, 1977), Lecture Notes in Math., vol. 673, Springer-Verlag, Berlin and New York, pp. 88-92.

[GHS] H. Glover, W. Homer and R. Stong, Splitting the tangent bundle of projective space, Indiana Univ. Math. J. (to appear).

[HMR] P. Hilton, G. Mislin and J. Roitberg, Localization of nilpotent groups and spaces, Math. Studies No. 15, North-Holland, Amsterdam, 1975.

[L1] A. Liulevicius, Homotopy rigidity of linear actions: characters tell all, Bull. Amer. Math. Soc. 84 (1978), 213-221.

[L2] _ Line bundles, cohomology automorphisms, and homotopy rigidity of linear actions, (Proc. Northwestern Univ. Conf. Geometric Applications of Homotopy Theory), Lecture Notes in Math., vol. 658, Springer-Verlag, Berlin and New York, 1978, pp. 224-233.

[L3] _ Flag manifolds and homotopy rigidity of linear actions, Algebraic Topology (Proc. Conf., Vancouver, 1977), Lecture Notes in Math., vol. 673, Springer-Verlag, Berlin and New York, 1978, pp. 254-261.

[O] L. O'Neill, The fixed point property for Grassmann manifolds, Ph.D. Dissertation, Ohio State Univ., Columbus, 1974.

[S] D. Sullivan, Infinitesimal computations in topology, Inst. Hautes Études Sci. Publ. Math. No. 47 (1977), 269-332. 